\title{
The distribution of freshwater gastropods on four Vanuatu islands : Espiritu Santo, Pentecost, Éfate and Tanna (South Pacific)
}

\author{
A. Haynes ${ }^{1}$
}

Keywords : Pacific, Vanuatu, freshwater, gastropods, distribution.

Streams and rivers on the Vanuatu islands of Espiritu Santo, Pentecost, Éfate and Tanna were sampled for gastropods and the physical and chemical characteristics of the water were investigated between 25/11/95 and 13/12/95. Twenty three species of neritid and forteen of thiarid prosobranch gastropods were found, together with the pulmonate Physastra nasuta and an opisthobranch species Strubellia. Six thiarid, two Clithon and one Neritina species, which are present in the Solomon Islands and South East Asia, but which are absent further south on New Caledonia and the Fiji islands, were found on the Vanuatu islands. On the other hand, Septaria bougainvillei and Clithon pritchardi, which are present on the Fiji islands and New Caledonia, were found no further north than the southern Vanuatu island of Tanna. This suggests that Vanuatu is a transition zone for both northern and southern freshwater snails. Éfate had many more species (26) than the other islands (16-19-. This was partly explained by its central geographical position and the higher ion content of its streams.

La distribution de Gastéropodes d'eau douce dans quatre îles de Vanuatu : Espiritu Santo, Pentecoste, Éfate et Tanna (Pacifique Sud)

Mots-clés : Pacifique, Vanuatu, eau douce, Gastéropodes, distribution.

Les Gastéropodes des cours d'eau de 4 îles de l'archipal de Vanuatu, Espiritu Santo, Pentecoste. Éfate et Tanna ont été échantillonnées et les caractéristiques physico-chimiques des milieux étudiées du 25/11/95 au 13/12/95. Vingt-trois espèces de néritidés et quatorze de gastéropodes prosobranches thiaridés ont été trouvés, avec le pulmonate Physastra nasuta et une espèce opistobranche de Strubellia. Six thiaridés, deux Clithon et une espèce de Neritina, qui sont présentes aux îles Solomon et en Asia du sud-est, mais absentes plus au sud en Nouvelle-Calédonie et aux Fidji, ont été trouvés à Vanuatu. En revanche, Septaria bougainvillei et Clithon pritchardi, qui sont présentes aux îles Fidji et en Nouvelle-Calédonie, n'ont pas été trouvés au nord de l'île méridionale Tanna de Vanuatu. Cela suggère que le Vanuatu est une zone de transition aussi bien pour les gastéropodes septentrionaux que méridionaux. Éfate possède beaucoup plus d'espèces (26) que les autres îles (15-19). Ceci s'explique, en partie, par sa position géographique centrale et la plus forte teneur en ions de ses rivières.

\section{Introduction}

Land and some freshwater molluscs of Vanuatu (ten New Hebrides) were reviewed by Solem (1959). He mentioned only three species of freshwater gastropods,

1. Institute of Applied Sciences, University of the South Pacific, P.O. Box 1168, Suva, Fiji.

Fax (679) 300373
Gyraulus montrouzieri (Gassies), Physastra nasuta (Morelet) and Fluviopupa brevior (Ancey), all found on Espiritu Santo. In his review he did not include the families Neritidae and Thiaridae. Nevertheless, many species of these families e.g. Neritina pulligera, Septaria suffreni and Thiara scabra were later found more than $20 \mathrm{~km}$ inland on Viti Levu, Fiji (Haynes 1985).

Starmühlner (1976) investigated some rivers (6 sites) on the island of Éfate and found 18 species including 6 species of thiarids, 10 species of neritids and $P$. nasuta and $F$. brevior. 
Vanuatu is a $Y$ shaped archipelago of 74 inhabited islands and over 200 smaller islands. In this study the streams and rivers of four islands were investigated. The islands were Espiritu Santo, the most northerly and largest, Pentecost to its south east, Éfate about $170 \mathrm{~km}$ further south and Tanna $200 \mathrm{~km}$ south of Éfate (Fig. 1).

Vanuatu's nearest neighbours are Solomon Islands $540 \mathrm{~km}$ to the north, New Caledonia $329 \mathrm{~km}$ to the south west and Fiji $600 \mathrm{~km}$ to the east (Fig. 1). The freshwater gastropods of the island groups, North and South and South of Vanuatu, had been investigated. Haynes (1993) studied the Solomon Islands of New Georgia, Guadalcanal, Mailata Makira and Haynes $(1985,1988 a, 1990)$ recorded the snails on most high islands of Fiji. Franc (1956), Starmühlner (1976) and Pöllabauer (1986) recorded freshwater snails in New Caledonia.

The previous investigations showed that certain gastropods are present in Solomon Islands but absent from
New Caledonia or Fiji, while others are present in New Caledonia or Fiji but absent from Solomon Islands.

As it is generally believed that neritid and thiarid gastropods arose in South East Asia (Starmühlner 1976, Haynes 1988b, 1990) and were distributed southwards into the south Pacific, it was anticipated that Vanuatu, situated between these three island groups, might be a region of faunal change. To test this eventuality, Vanuatu islands were sampled along a NorthSouth gradient.

\section{Study area}

The islands of Vanuatu are composed of mainly volcanic rock often overlaid by younger limestone. The East of Espiritu Santo is composed of recent limestone and has few streams while the western part is made of older volcanic rock but is inaccessible by road from Luganville. Sampling sites on Espiritu Santo were in the south and interior of the island (Fig. 2 A).

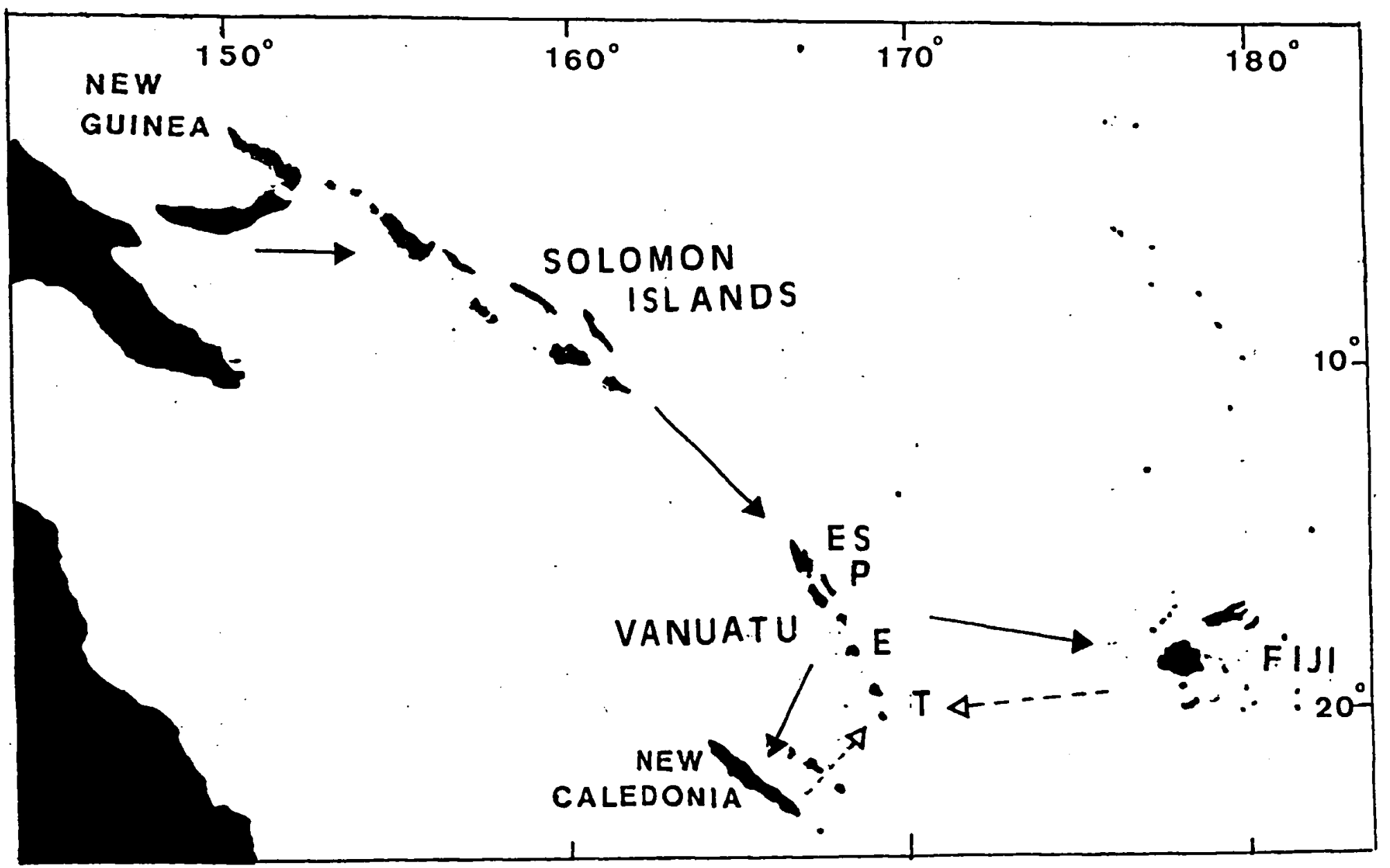

Fig.1. Map of the South Pacific. Arrows show the direction of dispersal of freshwater neritid and thiarid gastropods from South East Asia and the dotted arrows the possible dispersal to Tanna from New Caledonia and Fiji. ES : Espiritu Santo, P : Pentecost, E : Éfate, $\mathrm{T}$ : Tanna.

Fig. 1.-Carte du Pacifique sud-ouest. Les flèches indiquent la direction de répartition des néritidés et thiaridés Gastéropodes d'eau douce à partir de l'Asie du sud-est, et les flèches en pointillés, la dispersion póssible vers Tanna à partir de la Nouvelle-Calédonie et des Fidji. ES : Espiritu Santo, P : Pentecoste, E : Éfate, T : Tanna. 
A

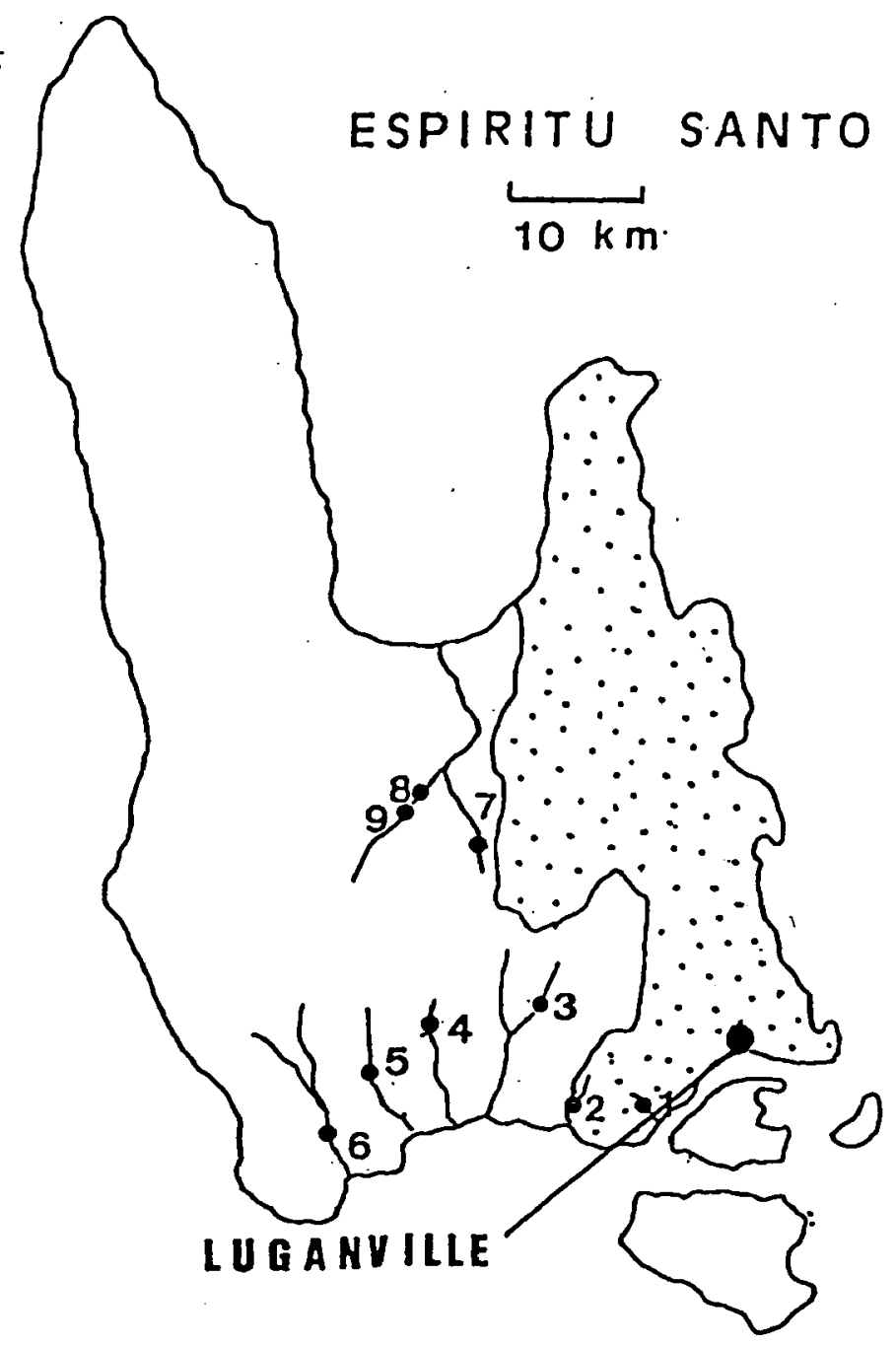

B

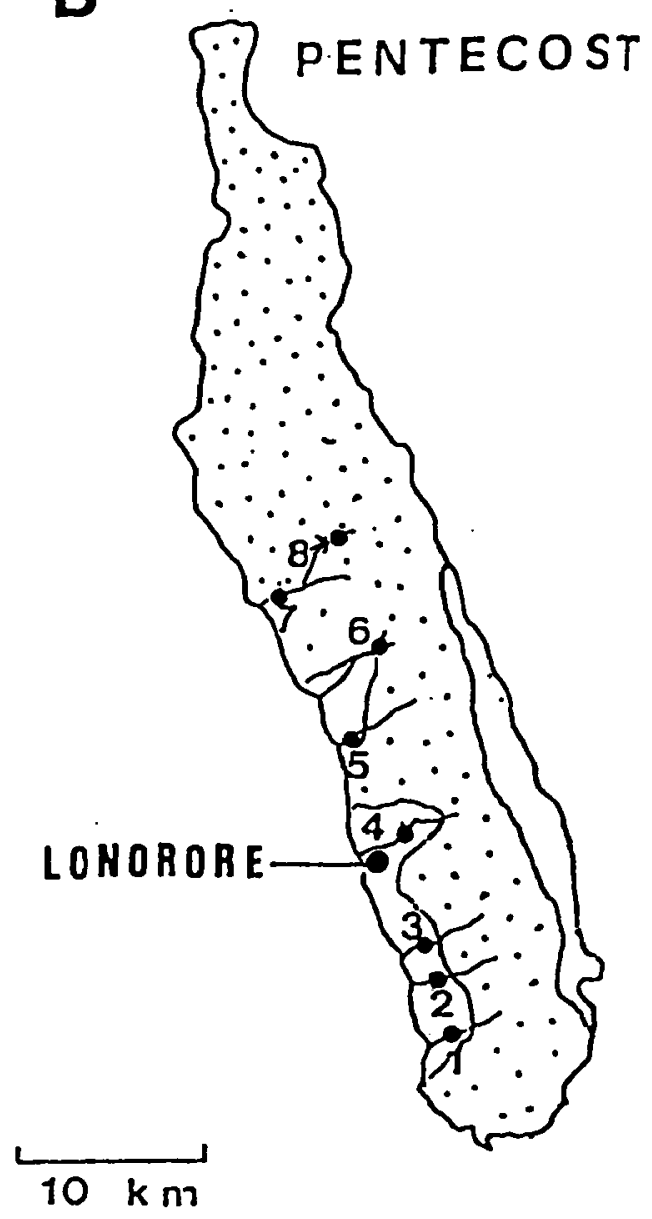

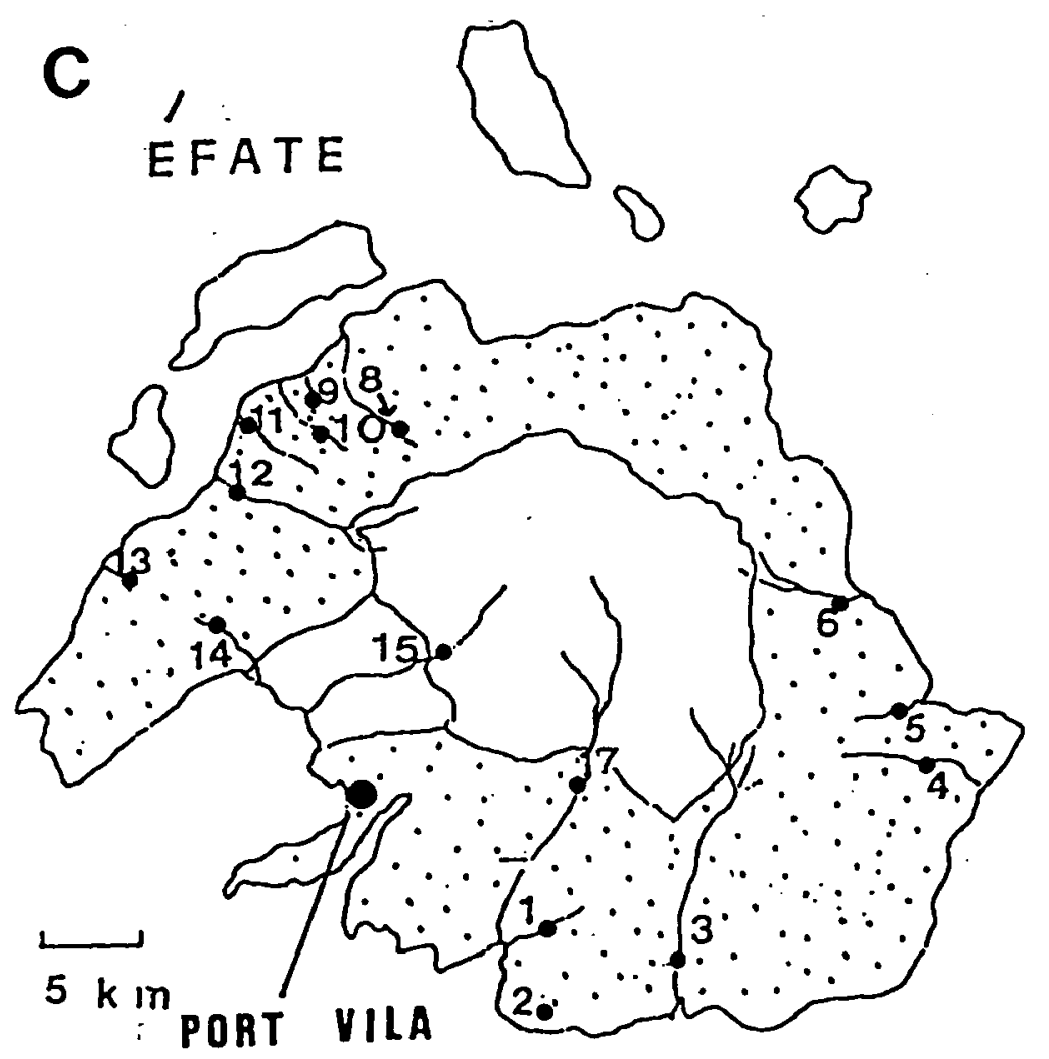

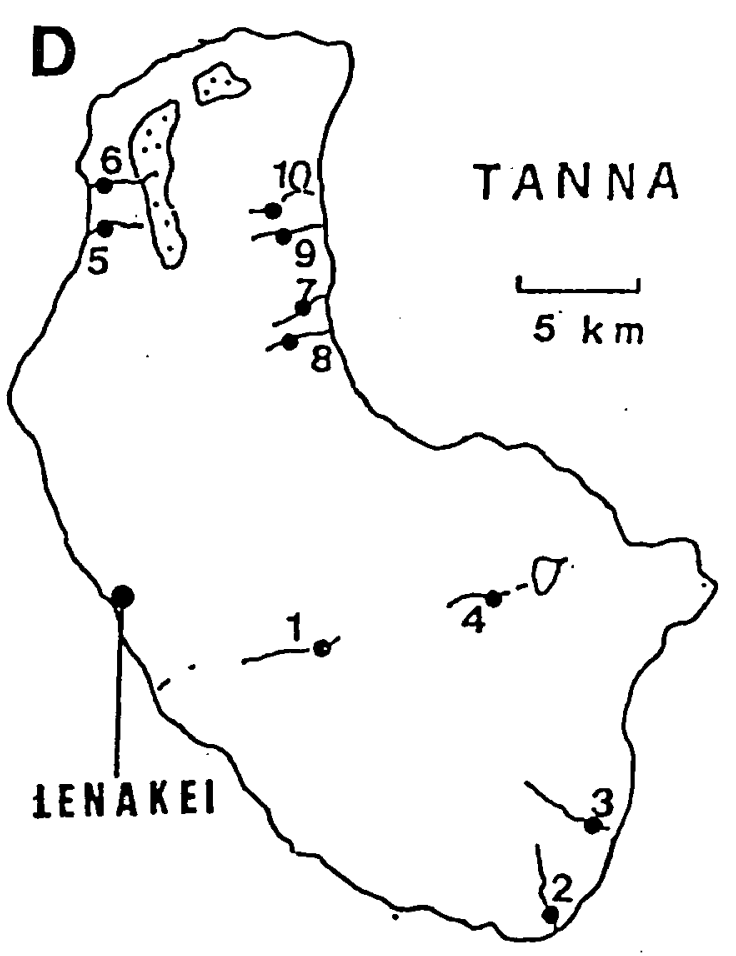

Fig. 2. Maps of the four Vanuatu islands_showing the_sampling-sites._Limestone-areas-are-stippled-

Fig. 2. Carte des quatre îles de Vanuatu, indiquant les sites d'échantillonnages. Les zones claires sont en pointillés. 
North Pentecost consists mainly of limestone overlaying older volcanic rocks and lacks permanent streams. The eastern side is very wet and inaccessible by road. Sampling sites were on the South East side where the substratum was mainly volcanic or vocanic rock overlaid by limestone (Fig. 2 B).

Éfate's rock is mainly pumice breccia overlaid by limestone. The road around the island made it possible to sample all coasts and inland at Lolima cascades (Fig. $2 \mathrm{C}$ : site 7) on the Teowma River.

Tanna's rocks are mainly volcanic rufts, breccias and basalts with a recently raised reef along the West coast where streams, between Lenakei and Kamera, were dry on the coast but still flowing in the hills (Fid. $2 \mathrm{D}$ : sites $1 \& 4)$.

\section{Material and methods}

Sampling took place on 25-28 November (Éfate), 2-5 December (Pentecost), 8-11 December (Espiritu Santo) and 11-12 December (Tanna).

Two or more people searched the substratum at each site for at least 30 minutes. Leaf litter, plants, wood and all surfaces of stones and boulders were inspected and sand and gravel were sieved. The specimens collected were killed in magnesium sulphate solution and preserved in $80 \%$ ethanol.

Water velocity and temperature were noted. Water samples collected at each site, were analysed for $\mathrm{pH}$ and total ions (conductivity $\mathrm{mS} \mathrm{m}^{-1}$ ) with sensormeters.

\section{Results}

The gastropod species found at each site on the four islands are given in Tables 1-4. A total of 39 species of gastropods were collected. Voucher specimens of the gastropods collected have been deposited in the Australian Museum, Sydney. The 23 species of Neritidae were Clithon bicolor (Récluz)(C.204240), C. chlorostoma (Sowerby)(C.204241), C. corona (Linné)(C.204 242), C. diadema, (Récluz)(C.204243), C. nucleolus (Morelet)(C.204244), C. olivaceus (Récluz)(C.204245), C. pritchardi (Dohrn)(C.204246), C. squarrosus (Récluz)(C.204247), Neritilia rubida (Pease)(C.204248), Neritina adumbrata (Reeve)(C.204249), N. asperulata Récluz(C.204250), N. auriculata Lamarck(204251), $N$. canalis Sowerby(C.204252), N. petitii Récluz (C.204253), N. porcata Gould(C.204254), N. pulligera Linné(C.204255), N. squamaepicta Récluz(C.204 256), $N$. tahitiensis Lesson(C.204257), N. turtoni Récluz(C.204258), N. variegata (Lesson)(C.204259),
Septaria bougainvillei (Récluz)(C.204260), S. porcellana (Linné)(C.204261), S. sanguisuga (Reeve)(C.204 262). The 14 Thiaridae species were Balanocochlis glans (v.d.Busch)(C.204263), Melanoides arctecara Mousson(C.204264), M. aspirans (Hinds)(C.204265), $M$. costata Quoy \& Gaimard(C.204266), M. pallens (Reeve)(C.204267), M. plicaria (Born)(C.204268), M. punctata (Lamarck)(C.204269), M. torulosa (Bruguiere)(C.204270), M. tuberculata (Müller)(C.204271), Melanopsis frustulum Morelet(C.204272), Tarebia granifera (Lamarck)(C.204273), T. bellicosa (Hinds)(C.204274), T. cancellata Roding(C.204275), T. scabra (Müller)(C.204276). The other two species were the Planorbidae Physastra nasuta (Morelet)(204277) and an opisthobranch of the genus Strubellia (Kuthe)(204278).

The number of species found at each site varied from 1 to 12 (Tables $1-4$ ). The mean water velocity and the mean total ions were calculated to find if there was any correlation between either and the number of species present. The correlation coefficient $r=0.409$ for the number of species and water velocity was not significant, but $\mathbf{r}=0.698$ for the number of species and total ions indicated a correlation at the $5 \%$ level. Total ion content at some sites, that were less than $0.3 \mathrm{~km}$ from the sea, was influenced by tidal sea water.

Fig. 3 shows the distribution of neritid freshwater gastropod species and Fig. 4 of the thiarids throughout the four Vanuatu islands and the adjacent archipelagoes of Solomon Islands, New Caledonia and Fiji. Species present in Solomon Islands are also found in South East Asia (Reich 1937, Starmühlner 1976).

The neritids $N$. asperulata, $C$. bicolor, $C$. nucleulus and $S$. porcellana are not present in Fiji while $C$. squarrosus, $C$. chlorostoma and $N$. adumbrata are absent from both New Caledonia and Fiji. On the other hand, $C$. pritchardi and $S$. bougainvillei, present on New Caledonia and Fiji were only found on the most southerly Vanuatu island Tanna (Fig. 3) and they have not been reported from any island further north.

The thiarids also show a disruptive distribution (Fig. 4). M. aspirans and the worldwide species $M$. tuberculata are the only species found in all four countries. Six species ( $T$. granifera, $T$. cancellata, $M$. punctata, $M$. pallens, $M$. torulosa, $B$. glans) have not been distributed further South than Vanuatu although T. granifera is present in the eastern Pacific on the Society Islands (Pointier \& Marquet 1990).

The number of species found on each Vanuatu Island is not only determined by the island area and the number os streams present but also by the number of col- 
Table 1. Gastropods, water velocity and total ionic content at the Espiritu Santo island sites .

Tableau 1. Les Gastéropodes, vitesse de l'eau et contenu total en ions pour les sites d'Espiritu Santo.

\begin{tabular}{|c|c|c|c|c|c|}
\hline Site \& river & $\begin{array}{l}\text { Map } \\
\text { reference }\end{array}$ & $\begin{array}{l}\text { Distance } \\
\text { from sea } \\
(\mathrm{km})\end{array}$ & $\begin{array}{l}\text { Water } \\
\text { speed } \\
\left(\mathrm{m}^{-1} \mathbf{s}^{-1}\right)\end{array}$ & $\begin{array}{l}\text { Total } \\
\text { ions } \\
\left(\mathrm{m} \mathrm{S}^{-1}\right)\end{array}$ & Gastropods \\
\hline $\begin{array}{l}\text { 1. Stream on } \\
\text { south coast }\end{array}$ & $\begin{array}{l}15^{\circ} 34^{\prime} \mathrm{S} \\
167^{\circ} 7^{\prime} \mathrm{E}\end{array}$ & 0.3 & 0.1 & 54.5 & $\begin{array}{l}\text { N. auriculata, } N \text {. rubida, } \\
\text { C. oliveaceus, } T \text {. cancellata. }\end{array}$ \\
\hline 2. Road ford & $\begin{array}{l}15^{\circ} 36^{\prime} \mathrm{S} \\
167^{\circ} 4^{\prime} \mathrm{E}\end{array}$ & 0.2 & 0.3 & 45.4 & C. bicolor, M. punctata, T. scabra. \\
\hline 3. Adson R. & $\begin{array}{l}15^{\circ} 33^{\prime} \mathrm{S} \\
166^{\circ} 57^{\prime} \mathrm{E}\end{array}$ & 5.0 & 0.4 & 27.2 & $\begin{array}{l}\text { C. bicolor, C. squarrosus, } \text { M. punctata } \\
\text { C. olivaceus, M. plicaria, B. glans, } \\
\text { N. pulligera. }\end{array}$ \\
\hline 4. Wailapa $R$. & $\begin{array}{l}15^{\circ} 32^{\prime} \mathrm{S} \\
166^{\circ} 55^{\prime} \mathrm{E}\end{array}$ & 5.0 & 0.6 & 29.3 & $\begin{array}{l}\text { C. bicolor, C. olivaceus, } S \text {. porcellana, } \\
\text { M. punctata, M. torulosa, T. scabra. }\end{array}$ \\
\hline 5. Baialo R. & $\begin{array}{l}15^{\circ} 34^{\prime} \mathrm{S} \\
166^{\circ} 53^{\prime} \mathrm{E}\end{array}$ & 2.0 & 0.6 & 31.3 & $\begin{array}{l}\text { S. bicolor, C. squarrosus, C. olivaceus, } \\
\text { N. rubida, M. punctata, M. aspirans, } \\
\text { M. plicaria, B. glans, T. scabra, } \\
\text { T. granifera }\end{array}$ \\
\hline 6. Navaka $R$. & $15^{\circ} 36^{\prime} \mathrm{S}$ & 1.0 & 0.8 & 25.2 & - \\
\hline 7. Lape $\mathrm{R}$. & $\begin{array}{l}15^{\circ} 17^{\prime} \mathrm{S} \\
166^{\circ} 55^{\prime} \mathrm{E}\end{array}$ & 15.0 & 0.4 & 31.3 & $\begin{array}{l}\text { M. tuberculata, M. torulosa, T. scabra, } \\
\text { T. granifera, T. bellicosa }\end{array}$ \\
\hline 8. Ora R. & $\begin{array}{l}15^{\circ} 16^{\prime} \mathrm{S} \\
166^{\circ} 53^{\prime} \mathrm{E}\end{array}$ & 13.0 & 0.4 & 26.4 & $\begin{array}{l}\text { M. tuberculata, T. granifera, P. nasuta, } \\
\text { T. bellicosa. }\end{array}$ \\
\hline 9. Pool by Ora R. & $\begin{array}{l}15^{\circ} 16^{\prime} S \\
166^{\circ} 53^{\prime} \mathrm{E}\end{array}$ & 13.0 & 0 & 33.3 & T. granifera, T. bellicosa, $P$. nasuta. \\
\hline
\end{tabular}

Table 2. Gastropods, water velocity and total ionic content at the Pentecost island sites.

Tableau 2. Les Gastéropodes, vitesse de l'eau et contenu total en.ions pour les sites de Pentecoste.

\begin{tabular}{|c|c|c|c|c|c|}
\hline Site \& river & $\begin{array}{l}\text { Map } \\
\text { reference }\end{array}$ & $\begin{array}{c}\text { Distance } \\
\text { from sea } \\
(\mathrm{km})\end{array}$ & $\begin{array}{l}\text { Water } \\
\text { speed } \\
\left(\mathrm{m} \mathrm{s}^{-1}\right)\end{array}$ & $\begin{array}{c}\text { Total } \\
\text { ions } \\
\left(\mathrm{m} \mathrm{S}^{-1}\right)\end{array}$ & Gastropods \\
\hline 1. Warbot R. & $\begin{array}{l}15^{\circ} 58^{\prime} \mathrm{S} \\
168^{\circ} 12^{\prime} \mathrm{E}\end{array}$ & 0.3 & 0.6 & 36.3 & $\begin{array}{l}\text { C. bicolor, } C . \text { squarrosus, } C \text {. oliveaceus, } \\
\text { N. variegata, M. tuberculata, T. granifera }\end{array}$ \\
\hline 2. Pambo R. & $\begin{array}{l}15^{\circ} 55^{\prime} \mathrm{S} \\
168^{\circ} 12^{\prime} \mathrm{E}\end{array}$ & 0.3 & 0.3 & 37.4 & $\begin{array}{l}\text { C. bicolor, } C \text {. corona, } C \text {. olivaceus, } \\
\text { C. canalis, M. plicaria, M. pallens }\end{array}$ \\
\hline 3. Walap R. & $\begin{array}{l}15^{\circ} 53^{\prime} S \\
168^{\circ} 11^{\prime} \mathrm{E}\end{array}$ & 0.3 & 0.5 & 35.3 & $\begin{array}{l}\text { C. bicolor, C. squarrosus, } C \text {. olivaceus, } \\
\text { S. porcellana, M. torulosa. }\end{array}$ \\
\hline $\begin{array}{l}\text { 4. Airport R. } \\
\text { at Lonorore }\end{array}$ & $\begin{array}{l}15^{\circ} 52^{\prime} \mathrm{S} \\
168^{\circ} 10^{\prime} \mathrm{E}\end{array}$ & 0.4 & 0.7 & 32.3 & $\begin{array}{l}\text { C. bicolor, } C \text {. squarrosus, } C \text {. olivaceus, } \\
\text { N. canalis, S. porcellana, M. punctata. }\end{array}$ \\
\hline 5. Whitewater $R$. & $\begin{array}{l}15^{\circ} 49^{\prime} \mathrm{S} \\
168^{\circ} 10^{\prime} \mathrm{E}\end{array}$ & 0.2 & 0.5 & 35.3 & \\
\hline 6. Waterfall R. & $\begin{array}{l}15^{\circ} 48^{\prime} \mathrm{S} \\
168^{\circ} 10^{\prime} \mathrm{E}\end{array}$ & 1.0 & 0.6 & 29.3 & M. tuberculata \\
\hline 7. Melsisi R. & $\begin{array}{l}15^{\circ} 45^{\prime} \mathrm{S} \\
168^{\circ} 8^{\prime} \mathrm{E}\end{array}$ & 0.3 & 0.5 & 29.3 & $\begin{array}{l}\text { C. bicolor, } C . \text { squarrosus, } C \text {. olivaceus, } \\
C . \text { chlorostoma, S. porcellana, } \\
\text { M. aspirans, M. plicaria, } M \text {. punctata, } \\
\text { M. tuberculata }\end{array}$ \\
\hline $\begin{array}{l}\text { 8. Melsisi R. } \\
\text { gorge }\end{array}$ & $\begin{array}{l}15^{\circ} 44^{\prime} \mathrm{S} \\
168^{\circ} 9^{\prime} \mathrm{E}\end{array}$ & 1.0 & 0.6 & 32.3 & $\begin{array}{l}\text { S. porcellana, } M . \text { tuberculata, } \\
M . \text { punctata, } M . \text { arctecara. }\end{array}$ \\
\hline
\end{tabular}


Table 3. Gastropods, water velocity and total ionic content at the Efate island sites .

Tableau 3. Les Gastéropodes, vitesse de l'eau et contenu total en ions pour les sites d'Éfate.

\begin{tabular}{|c|c|c|c|c|c|}
\hline Site \& river & $\begin{array}{l}\text { Map } \\
\text { reference }\end{array}$ & $\begin{array}{l}\text { Distance } \\
\text { from sea } \\
(\mathrm{km})\end{array}$ & $\begin{array}{l}\text { Water } \\
\text { speed } \\
\left(\mathrm{m} \mathrm{s}^{-1}\right)\end{array}$ & $\begin{array}{l}\text { Total } \\
\text { ions } \\
\left(\mathrm{m} \mathrm{S}^{-1}\right)\end{array}$ & Gastropods \\
\hline $\begin{array}{l}\text { 1. Small } \\
\text { stream }\end{array}$ & $\begin{array}{l}17^{\circ} 48^{\prime} \mathrm{S} \\
168^{\circ} 22^{\prime} \mathrm{E}\end{array}$ & 0.2 & 0.1 & 14.0 & N. tuberculata, M. costata, T. bellicosa \\
\hline $\begin{array}{l}\text { 2. Pond at White } \\
\text { Sands }\end{array}$ & $\begin{array}{l}17^{\circ} 49^{\prime} S \\
168^{\circ} 23^{\prime} \mathrm{E}\end{array}$ & 0.1 & 0 & 11.2 & M. costata. \\
\hline 3. Japanese R. & $\begin{array}{l}17^{\circ} 45^{\prime} \mathrm{S} \\
168^{\circ} 27^{\prime} \mathrm{E}\end{array}$ & 0.2 & 0.3 & 48.0 & $\begin{array}{l}\text { C. diadema, N. auriculata, T. scabra, } \\
\text { n. tahitiensis. }\end{array}$ \\
\hline 4. Ewor R. & $\begin{array}{l}17^{\circ} 42^{\prime} \mathrm{S} \\
168^{\circ} 33^{\prime} \mathrm{E}\end{array}$ & 0.5 & 0.4 & 50.0 & $\begin{array}{l}\text { C. nucleolus, } C \text {. squarrosus, } C \text {. corona, } \\
\text { N. variegata, } M \text {. tuberculata, } M \text {. plicaria. }\end{array}$ \\
\hline 5. Forari $\mathbf{R}$. & $\begin{array}{l}17^{\circ} 41^{\prime} \mathrm{S} \\
168^{\circ} 31^{\prime} \mathrm{E}\end{array}$ & 0.3 & 0.3 & 31.0 & M. plicaria, M. tuberculata. \\
\hline $\begin{array}{l}\text { 6. Tufala R. } \\
\text { at Epao }\end{array}$ & $\begin{array}{l}17^{\circ} 37^{\prime} \mathrm{S} \\
168^{\circ} 29^{\prime} \mathrm{E}\end{array}$ & 0.3 & 0.5 & 36.0 & $\begin{array}{l}\text { C. corona, C. diadema, C. squarrosus, } \\
\text { S. porcellana, M. plicaria, M. tuberculata }\end{array}$ \\
\hline $\begin{array}{l}\text { 7. Lolima falls } \\
\text { Taouma R. }\end{array}$ & $\begin{array}{l}17^{\circ} 43^{\prime} \mathrm{S} \\
168^{\circ} 24^{\prime} \mathrm{E}\end{array}$ & 5.0 & 0.5 & 48.0 & M. tuberculata, M. pallens \\
\hline $\begin{array}{l}\text { 8. La Marona R. } \\
\text { (N. West) }\end{array}$ & $\begin{array}{l}17^{\circ} 34^{\prime} \mathrm{S} \\
168^{\circ} 17^{\prime} \mathrm{E}\end{array}$ & 3.0 & 0.3 & 64.0 & $\begin{array}{l}\text { C. corona, } C . \text { diadema, } C . \text { nucleolus, } \\
\text { C. squarrosus, } N . \text { asperulata, } N . \text { porcata, } \\
\text { N. canalis, } N . \text { pulligera, } \\
\text { N. squamaepicta, M. plicaria, Strubellia } \\
\text { Sp., M. aspirans, T. bellicosa }\end{array}$ \\
\hline $\begin{array}{l}\text { 9. Outlet of } \\
\text { American } \\
\text { pool }\end{array}$ & $\begin{array}{l}17^{\circ} 35^{\prime} \mathrm{S} \\
168^{\circ} 16^{\prime} \mathrm{E}\end{array}$ & 0.5 & 0.3 & 62.0 & $\begin{array}{l}\text { N. adumbrata, } N . \text { variegata, } N . \text { rubida, } \\
\text { M. tuberculata, Melanopsis sp., } \\
\text { Strubellia sp. }\end{array}$ \\
\hline $\begin{array}{l}\text { 10. Sunai } \\
\text { stream }\end{array}$ & $\begin{array}{l}17^{\circ} 35^{\prime} \mathrm{S} \\
168^{\circ} 17^{\prime} \mathrm{E}\end{array}$ & 1.0 & 0.7 & 48.0 & N. adumbrata \\
\hline $\begin{array}{l}\text { 11. Stream at } \\
\text { Ulei school }\end{array}$ & $\begin{array}{l}17^{\circ} 35^{\prime} \mathrm{S} \\
168^{\circ} 16^{\prime} \mathrm{E}\end{array}$ & 0.1 & 0.3 & 56.0 & $\begin{array}{l}\text { C. corona, C. squarrosus, } N . \text { rubida, } \\
\text { N. variegata, M. aspirans, M. plicaria, } \\
\text { M. tuberculata, T. bellicosa }\end{array}$ \\
\hline 12. Ai creek & $\begin{array}{l}17^{\circ} 37^{\prime} \mathrm{S} \\
168^{\circ} 14^{\prime} \mathrm{E}\end{array}$ & 0.5 & 0.4 & 37.0 & $\begin{array}{l}C . \text { corona, } C \text {. squarrosus, } C \text {. chlorostoma, } \\
\text { N. petitii, N. variegata, } M \text {. tuberculata, } \\
S . \text { porcellana, M. punctata, } N \text {. rubida. }\end{array}$ \\
\hline $\begin{array}{l}\text { 13. Buria } \\
\text { village }\end{array}$ & $\begin{array}{l}17^{\circ} 38^{\prime} \mathrm{S} \\
168^{\circ} 12^{\prime} \mathrm{E}\end{array}$ & 0.2 & 0.5 & 44.0 & $\begin{array}{l}\text { C. corona, C. chlorostom, S. porcellana, } \\
\text { M. tuberculata. }\end{array}$ \\
\hline $\begin{array}{l}\text { 14. Cascades } \\
\text { stream }\end{array}$ & $\begin{array}{l}17^{\circ} 41^{\prime} \mathrm{S} \\
168^{\circ} 15^{\prime} \mathrm{E}\end{array}$ & 0.5 & 0.5 & 38.0 & $\begin{array}{l}\text { C. corona, } N . \text { petitii, } N \text {. variegata, } \\
M . \text { aspirans, } M . \text { punctata. }\end{array}$ \\
\hline 15. Savaroa R. & $\begin{array}{l}17^{\circ} 42^{\prime} \mathrm{S} \\
168^{\circ} 18^{\prime} \mathrm{E}\end{array}$ & 5.0 & 0.4 & 22.0 & - \\
\hline
\end{tabular}

lecting sites visited (Table 5). The number of species found on Effate increased sharply as the first sites were sampled but increased slowly after sampling 9 sites (Fig. 5). Twenty four (62\%) species had been found after 9 sites were sampled. The result would have been the same whether sampling occurred in an easterly or westerly direction from Port Vila. If site 8 had been missed, only $22(85 \%)$ of the total species would have been found (Fig. 5). However, as no less than eight sites were investigated on an island, the number of species found is not far short of the actual number present.

When the sites are classified according to distance from the sea, sites $6 \mathrm{~km}$ or more from the sea have four species of thiarids and $P$. nasuta only, while neritids have not colonized the rivers farther than $5 \mathrm{~km}$ from the sea (Table 6). 
Table 4. Gastropods, water velocity and total ionic content at the Tanna island sites.

Tableau 4. Les Gastéropodes, vitesse de l'eau et contenu total en ions pour les sites de Tanna.

\begin{tabular}{|c|c|c|c|c|c|}
\hline Site $\&$ river & $\begin{array}{l}\text { Map } \\
\text { reference }\end{array}$ & $\begin{array}{l}\text { Distance } \\
\text { from sea } \\
(\mathrm{km})\end{array}$ & $\begin{array}{l}\text { Water } \\
\text { speed } \\
\left(\mathrm{m} \mathrm{s}^{-1}\right)\end{array}$ & $\begin{array}{l}\text { Total } \\
\text { ions } \\
\left(\mathrm{m} \mathrm{S}^{-1}\right)\end{array}$ & Gastropods \\
\hline $\begin{array}{l}\text { South Coast } \\
\text { 1. Below water- } \\
\text { fall Custom } \\
\text { village }\end{array}$ & $\begin{array}{l}19^{\circ} 36^{\prime} \mathrm{S} \\
169^{\circ} 22^{\prime} \mathrm{E}\end{array}$ & 5.0 & 0.4 & 21.1 & C. corona, M. tuberculata, $P$. nasuta. \\
\hline $\begin{array}{l}\text { 2. Stream } 1 \mathrm{~km} \\
\text { from Kwamera }\end{array}$ & $\begin{array}{l}19^{\circ} 39^{\prime} \mathrm{S} \\
168^{\circ} 24^{\prime} \mathrm{E}\end{array}$ & 0.1 & 0.3 & 16.1 & $\begin{array}{l}\text { C. corona, } C \text {. olivaceus, } S \text {. porcellana, } \\
\text { S. sanguisuga, } M \text {. tuberculata. }\end{array}$ \\
\hline $\begin{array}{l}\text { 3. Kwamera } \\
\text { stream }\end{array}$ & $\begin{array}{l}19^{\circ} 39^{\prime} \mathrm{S} \\
169^{\circ} 26^{\prime} \mathrm{E}\end{array}$ & 0.1 & 0.3 & 19.2 & $\begin{array}{l}\text { C. corona, } C \text {. olivaceus, } S \text {. porcellana, } \\
\text { C. squarrosus }\end{array}$ \\
\hline $\begin{array}{l}\text { 4. Iakaplalaken } \\
\text { str., Isaka }\end{array}$ & $\begin{array}{l}19^{\circ} 33^{\prime} \mathrm{S} \\
169^{\circ} 25^{\prime} \mathrm{E}\end{array}$ & 6.0 & 0.8 & 23.3 & P. nasuta \\
\hline $\begin{array}{l}\text { North Coast } \\
\text { 5. Lembo } \\
\text { stream }\end{array}$ & $\begin{array}{l}19^{\circ} 23^{\prime} \mathrm{S} \\
169^{\circ} 25^{\prime} \mathrm{E}\end{array}$ & 0.1 & 0.4 & 54.5 & $\begin{array}{l}\text { C. corona, C. squarrosus, } \\
\text { N. variegata, S. porcellana, } \\
\text { M. aspirans, M. plicaria }\end{array}$ \\
\hline $\begin{array}{l}\text { 6. Loanatanu } \\
\text { stream }\end{array}$ & $\begin{array}{l}19^{\circ} 22^{\prime} \mathrm{S} \\
169^{\circ} 14^{\prime} \mathrm{E}\end{array}$ & 0.1 & 0.3 & 49.5 & $\begin{array}{l}\text { C. corona, } C . \text { squarrosus, } C \text {. bicolor, } \\
\text { N. canalis, N. pulligera, N. variegata, } \\
\text { S. porcellana, S. bougainvillei, } \\
\text { M. punctata, M. aspirans }\end{array}$ \\
\hline $\begin{array}{l}\text { East } \frac{\text { Coast }}{7 . \text { Nowanatanu }} \\
\text { stream }\end{array}$ & $\begin{array}{l}19^{\circ} 26^{\prime} \mathrm{S} \\
169^{\circ} 20^{\prime} \mathrm{E}\end{array}$ & 0.1 & 0.3 & 30.3 & $\begin{array}{l}\text { C. corona, } C \text {. squarrosus, } N \text {. turtoni, } \\
\text { N. variegata, S. porcellan. }\end{array}$ \\
\hline $\begin{array}{l}\text { 8. Locknaula } \\
\text { stream }\end{array}$ & $\begin{array}{l}19^{\circ} 26^{\prime} \mathrm{S} \\
169^{\circ} 21^{\prime} \mathrm{E}\end{array}$ & 0.2 & 0.3 & 29.3 & $\begin{array}{l}\text { C. corona, } C \text {. olivaceus, } C \text {. bicolor, } \\
\text { S. porcellana, S. bougainvillei. }\end{array}$ \\
\hline $\begin{array}{l}\text { 9. Itnime } \\
\text { stream }\end{array}$ & $\begin{array}{l}19^{\circ} 25^{\prime} \mathrm{S} \\
169^{\circ} 20^{\prime} \mathrm{E}\end{array}$ & 0.2 & 0.4 & 38.4 & $\begin{array}{l}\text { C. corona, } C . \text { bicolor, } C \text {. pritchardi, } \\
\text { S. porcellana, S. bougainvillei, } \\
B . \text { glans, } M . \text { aspirans, } M . \text { pallens. }\end{array}$ \\
\hline $\begin{array}{l}\text { 10. Noh-keiu } \\
\text { stream }\end{array}$ & $\begin{array}{l}19^{\circ} 24^{\prime} \mathrm{S} \\
169^{\circ} 20^{\prime} \mathrm{E}\end{array}$ & 0.2 & 0.3 & 27.3 & $\begin{array}{l}\text { C. corona, } N . \text { variegata, } S . \text { porcellana, } \\
\text { S. sanguisuga, M. aspirans, M. plicaria, } \\
\text { B. glans. }\end{array}$ \\
\hline
\end{tabular}

Table 5. The number of freshwater gastropod species found, the areas and stream length of each island, the number of sites visited and range of $\mathrm{pH}$ and temperature for four Vanuatus islands.

Tableau 5. Le nombre de Gastéropodes d'eau douce trouvés, la superficie et longueur des ruisseaux, le nombre de sites visités et les valeurs du $\mathrm{pH}$ et de la température dans les quatre îles de Vanuatu.

\begin{tabular}{lcccccc}
\hline Island & $\begin{array}{c}\text { River \& stream } \\
\text { Length }(\mathrm{km})\end{array}$ & $\begin{array}{c}\text { Area } \\
\left(\mathrm{km}^{2}\right)\end{array}$ & $\begin{array}{c}\text { Number of } \\
\text { species }\end{array}$ & $\begin{array}{c}\text { Number of } \\
\text { sites }\end{array}$ & $\mathrm{pH}$ & $\begin{array}{c}\text { Temperature } \\
\left.{ }^{\circ} \mathrm{C}\right)\end{array}$ \\
\hline Espiritu Santo & 2300 & 6840 & 18 & 9 & $7.0-7.3$ & $23-29$ \\
Éfate & 370 & 900 & 26 & 15 & $7.0-7.8$ & $24-26$ \\
Éfate & & & & & & \\
(Starmühlner 1976) & 370 & 900 & 18 & 6 & $6.8-7.2$ & $23-27$ \\
Tana & 170 & 560 & 19 & 10 & $6.9-7.4$ & $22-27$ \\
Pentecost & 90 & 430 & 16 & 8 & $7.0-7.4$ & $23-27$ \\
\hline
\end{tabular}




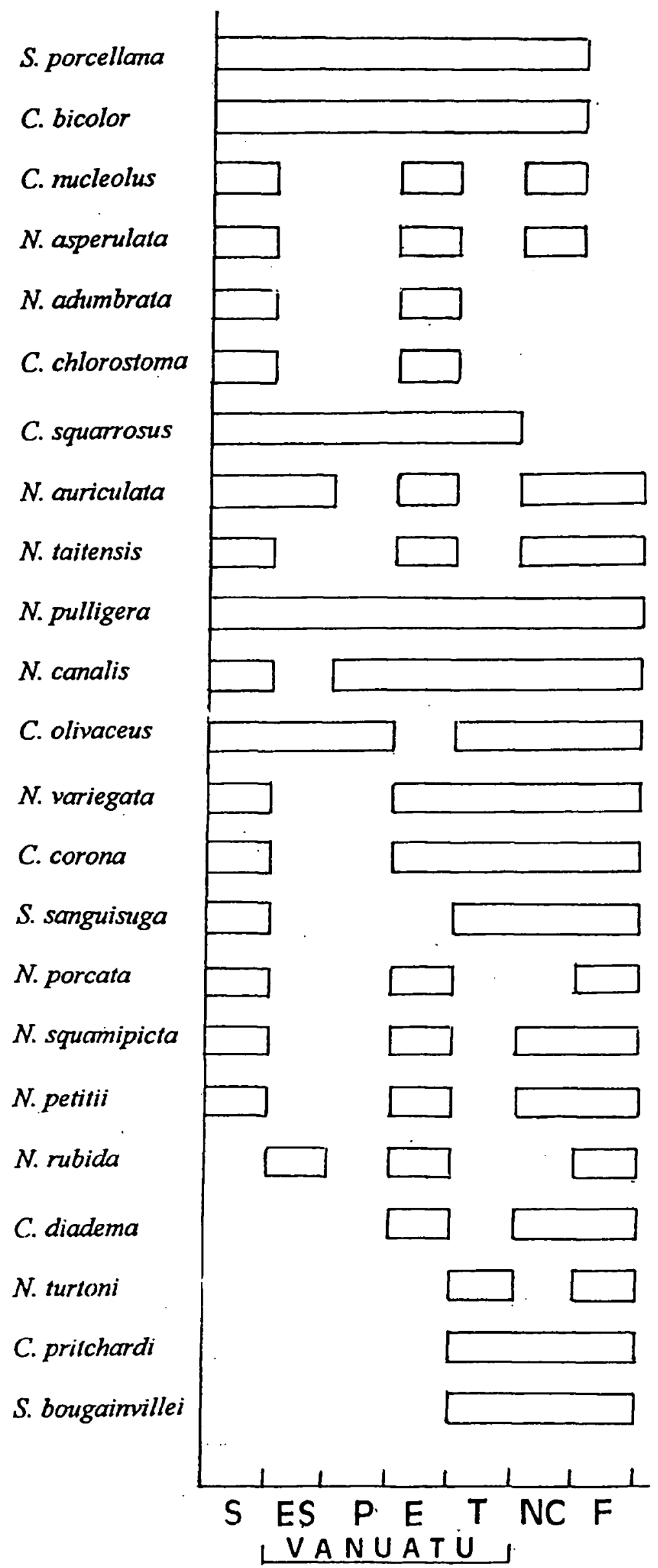

Fig.3. The distribution of freshwater neritid gastropod species on four Vanuatu islands. (ES : Espiritu Santo, P : Pentecost, E : Éfate, $\mathrm{T}$ : Tanna) and the adjacent island groups of Solomon Islands (S), New Caledonia (NC), and Fiji (F.).

Fig. 3. La distribution des espèces de Gastéropodes néritidés d'eau douce sur quatre îles de l'archipel de Vanuatu (ES : Espiritu Santo ; P : Pentecoste, E : Éfate, T : Tanna) et dans les archipels voisins des Salomon (S), Nouvelle-Calédonie (NC) et Fidji (F).

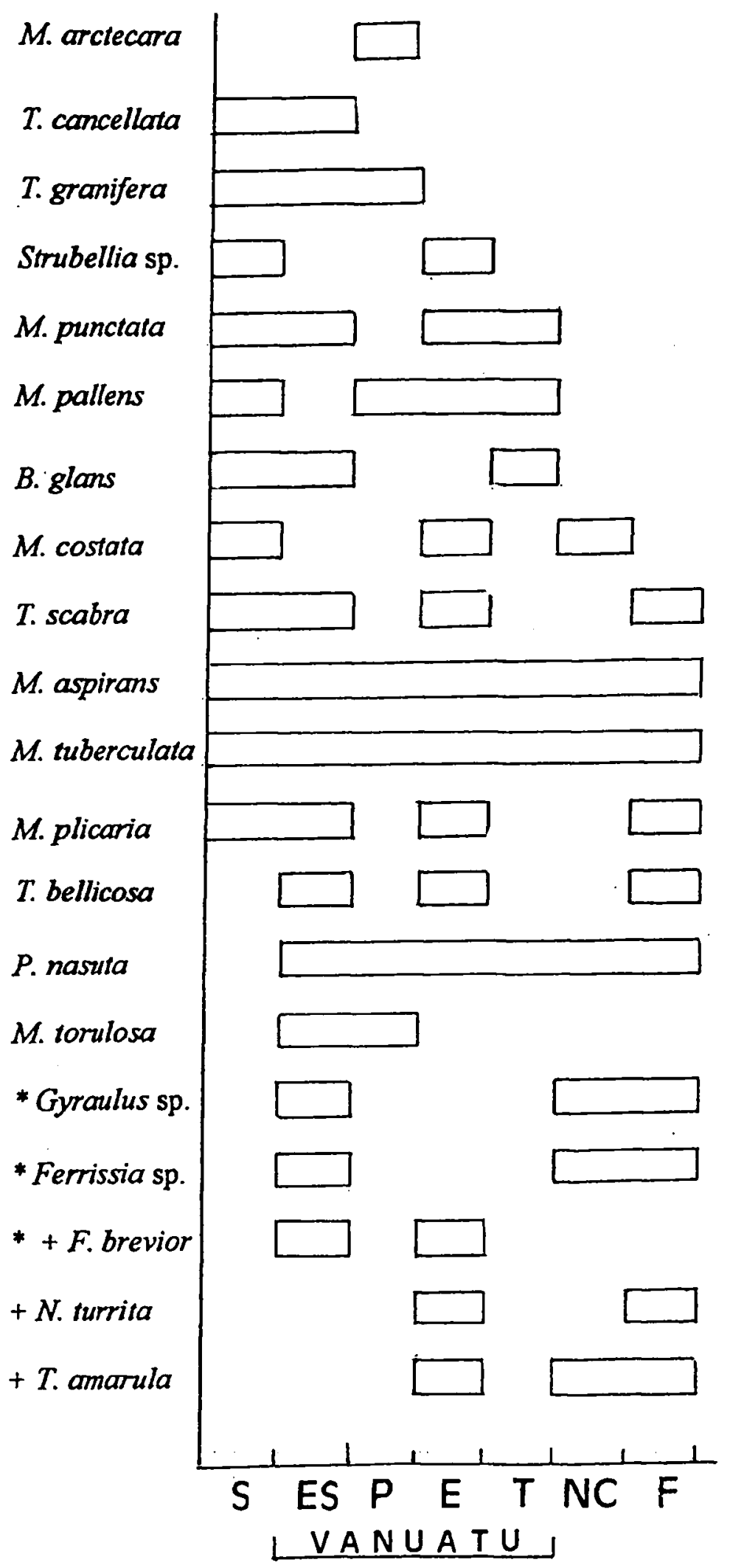

Fig.4. The distribution of freshwater thiarid and some gastropod species on four Vanuatu islands. (ES : Espiritu Santo, P : Pentecost, $\mathrm{E}:$ Éfate, $\mathrm{T}$ : Tanna) and on the adjacent groups of Solomon Islands (S), New Caledonia (NC), and Fiji (F.). * Solem (1959), + Starmühlner (1976).

Fig. 4. La distribution des thiaridés d'eau douce et autres espèces de Gastéropodes sur quatre îles de l'archipel de Vanuatu (ES : Espiritu Santo ; P : Pentecoste, E : Éfate, T : Tanna) et les archipels voisins des Salomon (S), Nouvelle-Calédonie (NC) et Fidji (F). 


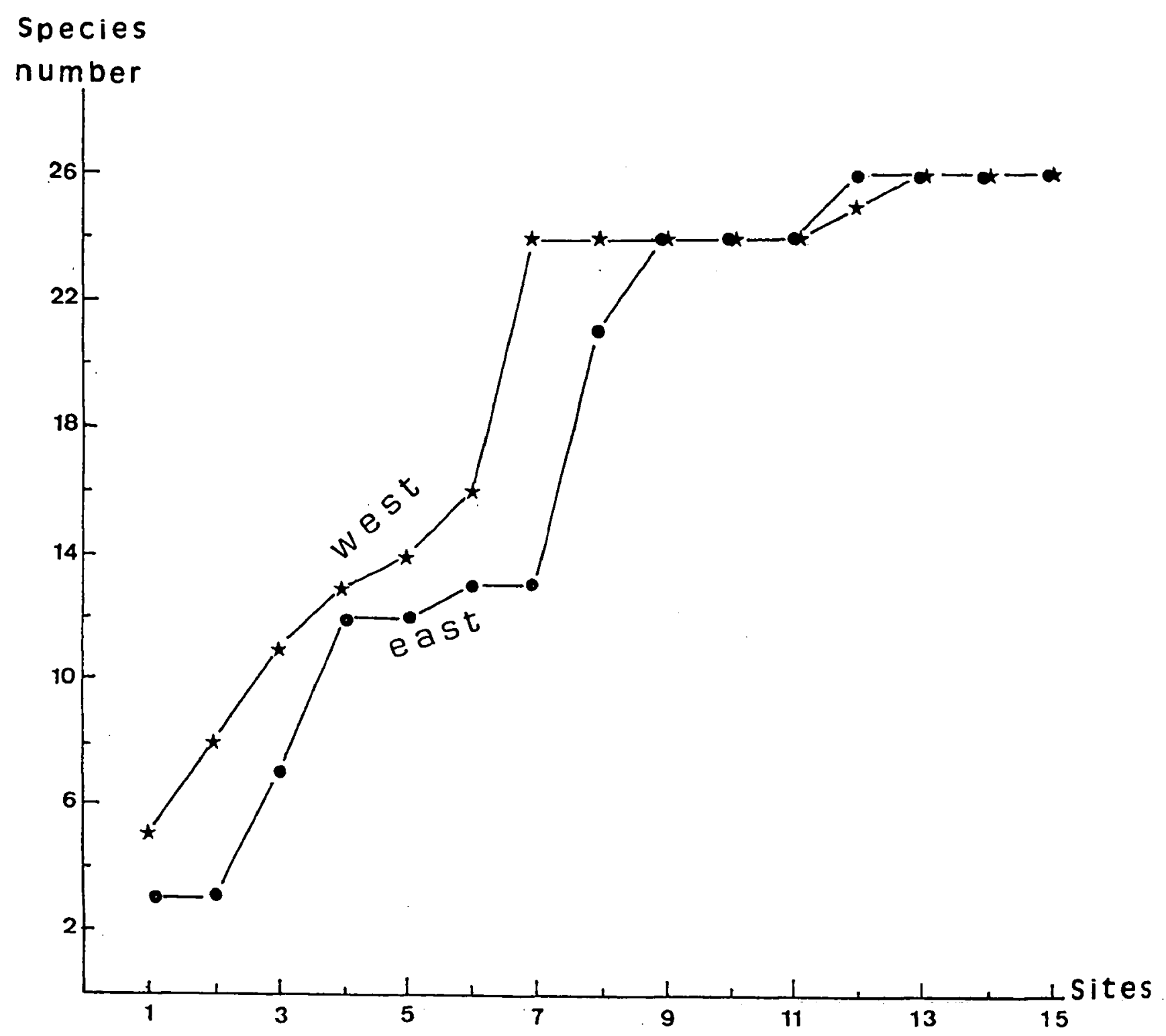

Fig. 5. The cumulative number of species found from sampling 15 sites in both easterly and westerly directions from Port Vila on Éfate.

Fig. 5. Nombre cumulé d'espèces découvertes en échantillonnant 15 sites dans des directions est et ouest à partir de Port Vila sur Éfate.

Shells of both neritids and thiarids were encrusted with lime where $\mathrm{CaCO}_{3}$, was high. This was noted on Pentecost at site 6, Waterfall R. and on Éfate at site 4, Ewor R. and site 7, Lololima Falls (Tables 2, 3).

\section{Discussion}

As well as the 39 species of gastropods recorded in this investigation, Solem (1959) found on Espiritu Santo the pulmonates Gyraulus montrouzieri and Ferrissia noumeensis previously recorded in slow moving water and ponds in Fiji and New Caledonia (Starmühlner 1970, Haynes 1985). Starmühlner (1976) found $F$. brevior, T. amarula and N. turrita on Éfate.

More species (26) were discovered on Éfate than on any of the other islands. Starmühlner (1976), who visited only $\overline{6}$ sites on Éfate found 17 species, compared with 18 species from 9 sites on Espiritu Santo and 19 species from 10 sites on Tanna in this study. The higher number of species on Éfate may be partly due to the general higher ion content of its waters. A weak correlation between total ions and species number was shown to exist. Other possible factors are its geographical position in the main line of any southward movement of gastropods, its composition of pumice breccia (not porous limestone as on other islands) and its relatively gentle slope near the coast.

Tanna, the most southerly island, had two neritid species, $S$. bougainvillei and C. pritchardi which were not found on other Vanuatu islands or further North in the Solomon Islands and South East Asia, but are common in New Caledonia (Pöllabauer 1986) and Fiji (Haynes_19.88a, 19.96,-19.9.7).-These-two-species-appear to have originated in this southern region. 
Table 6. The number of times each freshwater gastropod species was found at sites classified according to their distance from the sea.

Tableau 6. Nombre de récoltes de chaque espèce de Gastéropodes dans 12 sites répartis en fonction de leur éloignement de la mer.

Taxa. $\quad$ Distance from sea $(\mathbf{k m})$

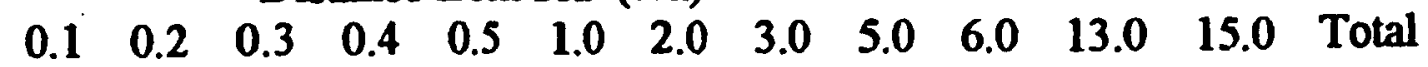

\begin{tabular}{|c|c|c|c|c|c|c|c|c|c|c|c|c|c|}
\hline Clithon bicolor & 1 & 3 & 4 & 1 & & & 1 & & & & & & 10 \\
\hline C. chlorostoma & & 1 & 1 & & & & & & & & & & 2 \\
\hline C. corona & 6 & 4 & 1 & & & & & 1 & 1 & & & & 13 \\
\hline C. diadema & & 1 & 1 & & & & & 3 & & & & & 5 \\
\hline C. nucleolus & & & & & 1 & & & 1 & & & & & 2 \\
\hline C. olivaceus & 2 & 1 & 5 & 1 & & & 1 & & 2 & & & & 12 \\
\hline C. pritchardi & 1 & & & & & & & & & & & & 1 \\
\hline C.squarrosus & 4 & & 4 & 1 & 2 & & 1 & 1 & 1 & & & & 14 \\
\hline Neritilia rubida & 1 & & 1 & & 1 & & 1 & & & & & & 4 \\
\hline Neritina adumbrata & & & & & 1 & 1 & & & & & & & 2 \\
\hline N. asperulata & & & & & & & & 1 & & & & & 1 \\
\hline N. auriculata & & 1 & 1 & & & & & & & & & & 2 \\
\hline N. canalis & 1 & & 1 & & & & & 1 & & & & & 3 \\
\hline$N \cdot$ petitii & & & & & 2 & & & & & & & & 2 \\
\hline N. porcata & & & & & & & & 1 & & & & & 1 \\
\hline N. pulligera & 1 & & & & & & & 1 & 1 & & & & 3 \\
\hline N. squamaepicta & & & & & & & & 1 & & & & & 1 \\
\hline N. tahitiensis & & 1 & & & & & & & & & & & 1 \\
\hline$N$. turtoni & 1 & & & & & & & & & & & & 1 \\
\hline N. variegata & 4 & 1 & 1 & & 4 & & & & & & & & 10 \\
\hline Septaria bougainvillei & 1 & 2 & & & & & & & & & & & 3 \\
\hline S. porcellana & 5 & 4 & 3 & 1 & 1 & 1 & & & 1 & & & & 16 \\
\hline S. sanguisuga & 1 & 1 & & & & & & & 1 & & & & 3 \\
\hline Balanocochlis glans & & 1 & & & & 1 & & & 1 & & & & 3 \\
\hline M.arctecara & & & & & & 1 & & & & & & & 1 \\
\hline M. aspirans & 2 & 2 & 2 & & 2 & & & & & & & & 8 \\
\hline M. costata & 2 & $\vdots$ & & & & & & & & & & & 2 \\
\hline M. pallens & & 1 & 1 & & & & & & & & & & 2 \\
\hline M. plicaria & 2 & 2 & 3 & & 1 & & 1 & 1 & 1 & & & & 11 \\
\hline$M$. punctata & 1 & 1 & 1 & 1 & 2 & 1 & 1 & & 2 & & & & 10 \\
\hline M. torulosa & & & 1 & & & & & & 1 & & & & 2 \\
\hline M. tuberculata & 2 & 3 & 3 & & 3 & 2 & & & 2 & & 1 & 1 & 17 \\
\hline Melanopsis frustulum & & & & & 1 & & & & & & & & 1 \\
\hline Tarebia granifera & & & 1 & & & & 1 & & & & 2 & 1 & 5 \\
\hline Thiara cancellata & & & 1 & & & & & & & & & & 1 \\
\hline T. bellicosa & 2 & & & & & & & & & & 2 & & 4 \\
\hline T. scabra & & 2 & & & & & & & 1 & & & 1 & 4 \\
\hline Physastra nasuta & & & & & & & & & 1 & 1 & 2 & & 4 \\
\hline Strubellia sp. & & & & & 1 & & & 1 & & & & & 2 \\
\hline Total & 40 & 32 & 36 & 5 & 22 & 7 & 7 & 13 & 16 & 1 & 7 & 3 & 189 \\
\hline
\end{tabular}


Vanuatu is as far South as many thiarid species have been distributed. The six species absent from both New Caledonia and Fiji may be poor dispersers, possibly because their young hatch as young adults rather than veligers or because the sometimes colder water temperatures : $16^{\circ} \mathrm{C}$ in New Caledonia (Starmühlner 1970 ) and $19^{\circ} \mathrm{C}$ in Fiji (Haynes 1985), has prevented them from becoming established.

Although the planorbid $P$. nasuta was present exclusively $5 \mathrm{~km}$ and more from the sea in Vanuatu, it lives in ponds and Sago swamps as near as one $\mathrm{km}$ from the sea on Viti Levu, Fiji (Haynes 1985). P. nasuta is not found in Solomon Islands but is present in New Caledonia (Starmühlner 1970), Tonga, Samoa and Cook Islands (Haynes 1990, Starmühlner 1993) as well as Fiji (Haynes 1985, 1988a). Other species of Physastra are found in Australia (Walker 1984).

Similarly, the genus Fluviopupa is a southern genus with endemic species in New Caledonia and Fiji as well as Vanuatu (Ponder 1982). It is not found further North than Vanuatu.

New Caledonia has at least two species of the thiarid, Melanopsis (Franc 1959, Starmühlner 1970) and one of these was present on Éfate. The opisthobranch, Strubellia sp., found in two streams on Éfate may be Strubellia paradoxa, which was found by Starmühlner (1976) on the island of Guadalcanal, Solomon Islands and which was discussed by Wawra $(1974,1988)$. The anatomy of the Vanuatu Strubellia has not been fully investigated.

\section{Acknowledgements}

I thank the University of the South Pacific for funds that made this work possible. I am also grateful for the help I received from Father Saprieu and the Sisters of Melsisi Catholic Mission, Pentecost and from students of USP Sub-Centre, Luganville, Espiritu Santo. In particular I thank USP Vanuatu students John Maki and Johnny Manango (Éfate) and Peter Pata (Tanna) for their cheerful and invaluable assistance. My thanks also to Dr. Antoine N'Yeurt for his help with the French text.

\section{References}

Franc A. 1956. - Mollusques terrestres et fluviatiles de l'Archipel Néo-Calédonien. Mémoires Mus. Nation. Hist. Nat., ser. A, Zoologie, $13: 1-200$.
Haynes A. 1985. - The ecology and local distribution of non-marine aquatic gastropods in Viti Levu, Fiji. The Veliger, 28 (2) : 204-210.

Haynes A. 1988a. - The gastropods in the streams and rivers of five Fiji islands (Vanua Levu, Ovalau, Gau, Kadavu and Taveuni. The Veliger, 30 (4) : 377-383.

Haynes A. 1988b - Notes on the stream neritids (Gastropoda ; Prosobranchia) of Oceania. Micronesica, $21: 93-102$.

Haynes A. 1990. - The number of freshwater gastropods on Pacific islands and the theory of island biogeography. Malacologia, 31 : 237-248.

Haynes A. 1993. - The gastropods in the streams and rivers of four islands (Guadalcanal, Makira, Malaita and New Georgia) in the Solomon Islands. The Veliger, 36 (3) : 285-290.

Haynes A. 1996. - Reproductive strategies in the freshwater genus Septaria (Neritidae). Molluscan Reproduction, Malacological Review, Suppl. $6: 1-7$.

Haynes A. 1997. - The distribution of five species of Septaria (Gastropoda : Neritoidea) in Fijian streams. The Veliger, 40 (1) : 71-76.

Pointier J.-P. \& Marquet G. 1990. - Taxonomy and distribution of freshwater mollusks of French Polynesia. Venus, 49 : 215-231.

Pöllabauer C. 1986. - Beitrag zur Taxonomie, Biologie und Ökologie mixohaliner polymorpher Neritiden (Archegastropoda : Mollusca). Doctoral Dissertation, Universitat Wien : 1-84.

Ponder W.F. 1982. - Hydrobiidae of Lord Howe Island (Mollusca : Gastropoda : Prosobranchia). Aust. J. Mar. Freshwater Res., 33 : 89-159.

Reich E. 1937. - Systematische, anatomische ökologische und tiergeographische Untersuchungen über die Süsswasser-Mollusken Papuasiens und Melanesiens. Arch. Fur Naturgeschichte (N.F.), $6: 37-153$.

Solem A: 1959. - Systematics and zoogeography of the land and fresh-water Mollusca of the New Hebrides. Fieldiana : Zoology, $43: 1-359$.

Starmühlner F. 1970. - Études hydrobiologiques en Nouvelle-Calédonie. O.R.S.T.O.M., sér. Hydrobiologie, 4 (3/4) : 3-127.

Starmühlner F. 1976. - Beiträge zur Kenntnis der Süsswasser-Gastropoden pazifischer Inseln. Ann. Naturhist. Mus. Wien., 80 : 473-656.

Starmühlner F. 1993. — Beiträge zur Kenntnis der Süsswasser- Gastropoden der Tonga-und Samoa-Inseln (SW-Pazifik). Ann. Naturhist. Mus. Wien, 80 : 94/95B : 217-306.

Walker J.-C. 1984. - Geographical relationships of the buliniform planorbids of Australia, In Solem A. \& A.C. Van Brugge, Worldwide snails : Biogeographical Studies on non-marine Mollusca. Brill/Backhuys, Leiden : 189-197.

Wawra E. 1974. - The rediscovery of Strubellia paradoxa (Strubell) (Gastropoda : Euthyneura : Acochlidiacea) on the Solomon Islands. The Veliger, 17 (1) : 8-10.

Wawra E. 1988. - Strubellia paradoxa (Strubell 1892) (Gastropoda : Opisthobranchia) von den Solomon-Inseln. Zool. Anz., 220 3/4:163-172. 